\title{
Grid-Style Modular Network Self-Organization Map for Detail Projection of Unknown Off-Shore Wind Speed
}

\author{
Mitsuharu Hayashi and Ken Nagasaka
}

\begin{abstract}
Wind generation is one of the fast growing and introduced resources among renewable energies through worldwide including Japan. As Japan, on the other hand, is an island country surrounded by ocean, the landscape topography suitable for wind generation is limited for the on-shore. Therefore, based on the wind map of up to year 2030, it is expected that new wind generation installation will be more suitable on off-shore rather than on-shore. For this reason, it is very important to determine the wind characteristics of the candidate area for installing wind generation, however in most cases of off-shore installation, existence of weather condition data is poor and needs lots of time and cost for measuring pin-point weather condition data. In this study, the goal of this research is to project a wind speed of an unseen area (where its weather condition data is not available) by mapping the seen areas (where their weather condition data are available) around the target area using the modularized Artificial Neural Network (SOM: Self-Organization Map). By learning the correlation between modularized ANNs of seen and unseen areas, the result of this temporal and spatial projection will be the prediction of wind speed of target place. Furthermore, in this study, by segmenting the area as grid-style and learning it, it becomes possible to predict the wind speed more detail and more precise. It is believed, by the help of the proposed technique, a huge amount of time and cost will be saved for selection of off-shore installation point of off-shore wind power generation. Moreover, it will certainly contribute to the development and speed-up of off-shore wind power generation in the future.
\end{abstract}

Index Terms-Artificial neural network, modular network som, projection, wind speed.

\section{INTRODUCTION}

One of the reasons that wind power generation has been spread in recent years is because the environmental problems such as greenhouse effect gas and global warming due to consumption of energy became worse than ever. IPCC (Intergovernmental Panel on Climate Change) mentioned that atmospheric level of greenhouse effect gas (i.e., carbon dioxide, methane, chlorofluocarbon, and the atmosphere of carbon monoxide) is increasing certainly by human activities, and there is a strong correlation between global warming with such greenhouse gas effects. It is also mentioned that the degree of carbon dioxide of contribution is the largest among all those greenhouse gas effects. The main artificial source of discharge of the carbon dioxide is consumption of fossil fuels Therefore, attention has been focused on the renewable

Manuscript received April 10, 2018; revised July 10, 2018.

Mitsuharu Hayashi and Ken Nagasaka are with the Department of Electronic and Information Engineering, Tokyo University of Agriculture and Technology, Japan (e-mail: takahayashi55@gmail.com, bahman@cc.tuat.ac.jp). energies as alternative energies to decrease consumption of fossil fuels in recent years. Wind energy is also the precious resources contributed to solution of global warming. [1], [2].

However, there are some disadvantages due to the characteristics of wind energy and landscape topography in on-shore of Japan as compared with other wind power high-introduced countries. In general, off-shore is known as better and suitable as for wind power generation, because the topography is less height and more flat on sea than angled landscapes on on-shore areas. In off-shore, wind speed and wind direction are more stable and the amount of power generation output is also stable. These issues will decrease the grid-connection stability problems to some extends.

As Japan is an island country surrounded by the sea, there is almost no factor that prevent the passage of wind at sea, therefore off-shore is suitable for wind power generation such as flat plains. Due to the NEDO (New Energy and Industrial Technology Development Organization of Japan) report on Wind Road Map up to year 2030, it is expected that new off-shore wind farms will be installed more on off-shore in the near future. [3]

One of the important factors necessary for selection of introduction point of wind power generation is proved to be the selection of wind conditions related to the candidate site. Obviously, the amount of power generation is reduced when unrelated weather condition information are used. Therefore, investigation of wind conditions before practical installation is required for optimal selection of the site.

Although it is important to determine the optimal point through some procedures similar to on-shore, however as for off-shore case, advance wind condition survey is difficult from the viewpoint of time and cost. Because, a pole has to be installed and fixed by cable to the bottom of the sea or in case of deep sea, it should be stand floating platform and collect data at least for 1-2 years for further weather condition analysis. The followings are the difficulties in detail:

- The existing weather data is few, so it is necessary to observe and collect weather condition data by providing a new technique for the candidate site.

- Installation of meteorological equipment and transfer data from off-shore (under the sea cabling, etc.) requires more cost compare with on-shore.

- For determination of the best installation site from the meteorological measurement point of view, it is necessary to measure plural points for the comparison.

- As a result of the weather observation, some cases abandon the introduction from the profitability, risk is inherent.

Based on the difficulties mentioned above, the 
break-through will be a way to find a technique to project weather condition data (for example wind speed in case of wind power generation) without direct measurement of meteorological data of the candidate site. By such technique, it becomes possible to reduce the cost and risk. In this research, our aim is to develop such this technique to project the wind condition of any points by using the other existing sites observation data. Furthermore, by segmenting the area more and learning it, it is possible to predict the wind speed in a more precise area.

\section{Wind SPEEd Projection TeChNIQUE}

In this study, two projections methods are used for wind speed projection as follows.

\section{A. Temporal Projection}

Wind speed is the data of time series change in a nonlinear behavior. Therefore, it is difficult to have a precious projection with traditional method because it requires a massive data. Some intelligent methods are proved to be effective for solving nonlinear problems, such as artificial neural network (ANN), genetic algorithms, deterministic chaos and so forth. These methods have been already utilized in many field's projection, such as stock price fluctuations, temperature rise of earth, electric load forecasting, etc.

In the past research of the authors of this paper, the ANN and deterministic chaos had confirmed that these methods are effective for the projection of wind speed [4], [5]. Fig. 1 shows the concept of temporal projection. Wind speed of future (unknown) is projected from the past historical data (known).

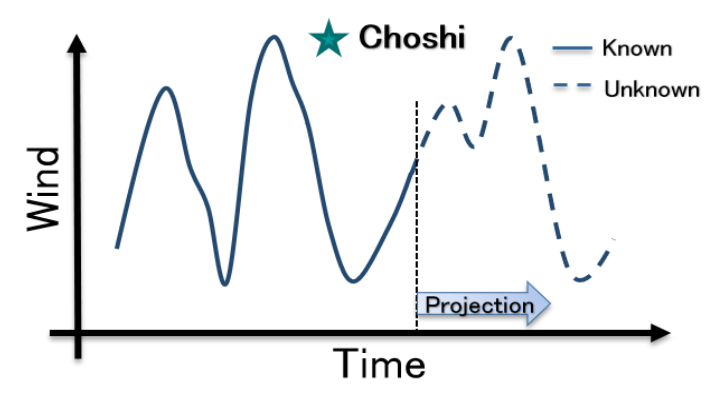

Fig. 1. Temporal projection.

\section{B. Spatial Projection}

As wind is caused by a difference in spatial atmospheric conditions and as the wind in continuous space is intended to be changed continuously, therefore, the changes of the displacement and wind speed are correlated. In the authors past research, the trends has confirmed that more information from neighborhood related to the wind direction of the target point make the projection result better.

In this research, this projection is combined the above two factors and by using the algorithm of modular network self-organization map (mnSOM) through some simulations, it improved the projection. The ANN, to project temporally for each point, is modularized and the spatial prediction is performed by learning the correlation between modules by update algorithm of the SOM.
Simulation data in this research are; wind speed and wind direction of the target point, called Choshi weather observatory, and 11 points around the target site. Fig. 2 shows the surrounding sits with observation data and the Choshi site with no available data which is used for projection.

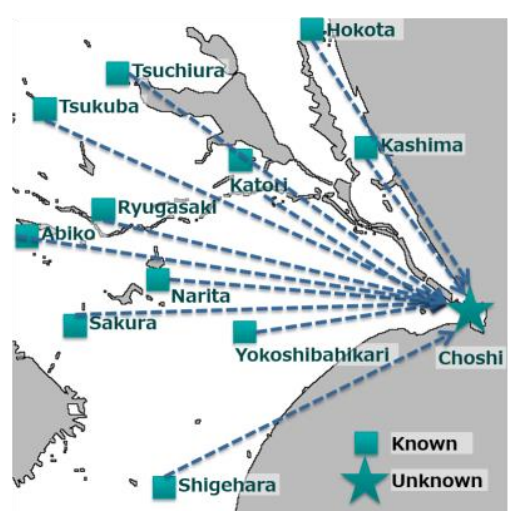

Fig. 2. Spatial projection.

\section{Simulation MethodS}

\section{A. Modular Network Self-Organization Map (mnSOM)}

The mnSOM is obtained by replacing each unit of Self-Organization Map (SOM) of Kohonen to modularized unit, such as ANN. In the case of using a multilayer perceptron which is one of the ANN as modular, it is known that the map is designed to have the properties near modular to each other by learning the different input and output correlation at each modules of mnSOM. The learned modular with given characteristic data are formed and show an intermediate output correlations. On the other hand, by forming interpolation, it is possible to learn the characteristics of modular which their data is not given [6]-[8].

Fig. 3 shows the construction and data flow of mnSOM.

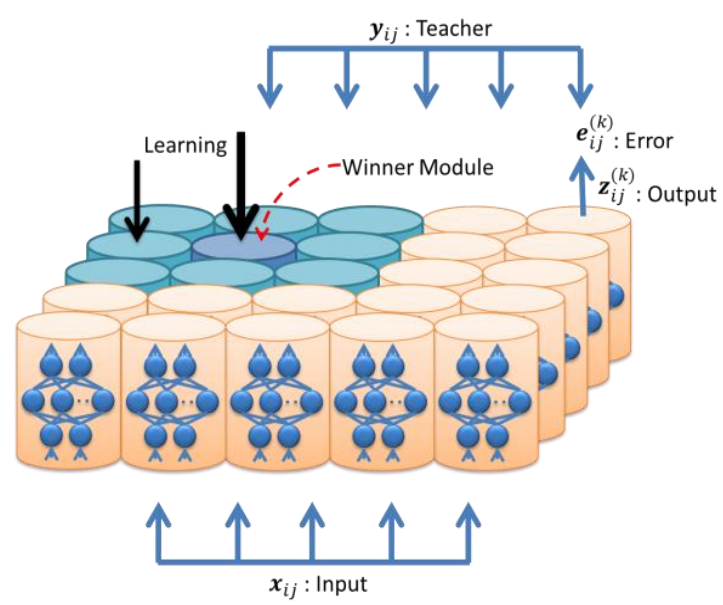

Fig. 3. Modular network SOM (mnSOM).

The basic learning algorithm of mnSOM is as follows.

First, it provides an input to all modules, leading to errors for each module from the output and the teacher signal.

Now, there are $\mathbf{M}$ system groups with $\mathbf{x}, \mathbf{y}$ as input and output vectors, and their input / output relations are modeled 
as $\mathbf{y}=f_{i}(\mathbf{x})(i=1,2, \ldots, M)$. The purpose of mnSOM is to let the module learn the input / output relation $f_{i}(\cdot)$ of each system based on the input / output dataset $D_{i}$, which is shown as following equation (1), by making the similarity between models into a self-organizing map.

$$
D_{i}=\left\{\left(\boldsymbol{x}_{i j}, \boldsymbol{y}_{i j}\right)\right\} \quad(i=1,2, \ldots, M)
$$

First, one set of $D_{i}$ can be selected randomly from the data group $D_{1}, \ldots, D_{M}$ of input and output, and also randomly select the input and output data $\left(\boldsymbol{x}_{i j}, \boldsymbol{y}_{i j}\right) \in D_{i}$ belonging to $D_{i}$. The $x_{i j}$ is an input of the network, given to all modules. The $\boldsymbol{y}_{i j}$ is the teacher (supervisor) signal. If $\boldsymbol{z}_{i j}^{(k)}$ is the output of the k-th module for the input $x_{i j}$, then the square error of the module is given by the following equation (2).

$$
e_{i j}^{(k)}=\frac{1}{2}\left\|\boldsymbol{y}_{i j}-z_{i j}^{(k)}\right\|^{2}
$$

Next, the winner module is determined based on the error of each module.

If $E_{i}^{(k)}$ shown in equation (3) is the total error of the k-th module for all data belonging to $D_{i}$, the module which minimizes the $E_{i}^{(k)}$ is the winner module as shown in equation (4).

$$
\begin{gathered}
E_{i}^{(k)}=\frac{1}{2} \sum_{j}\left\|y_{i j}-z_{i j}^{(k)}\right\|^{2}=\sum_{j} e_{i j}^{(k)} \\
k_{\text {winner }}\left(D_{i}\right)=\min _{k} E_{i}^{(k)}
\end{gathered}
$$

In the learning of mnSOM, actually the $E_{i}^{(k)}$ is replaced with the average error $\bar{E}_{i}^{(k)}$ of time to enable online learning and to speed up the decision of the winner. The average error $\bar{E}_{i}^{(k)}$ time is shown in equation (5), where $\varepsilon$ is a positive number close to zero.

$$
\bar{E}_{i}^{(k)}(t)=\varepsilon e_{i j}^{(k)}+(1-\varepsilon) \bar{E}_{i}^{(k)}(t-1)
$$

Learning is using the back-propagation method (BP), to update the connection weights of the winner and the neighboring modules. The weight $\boldsymbol{w}^{(k)}$ of the k-th module is updated by equation (6).

$$
\Delta \boldsymbol{w}^{(k)}(t)=\phi(t, l)\left(-\eta \frac{\partial E_{i}^{(k)}}{\partial \boldsymbol{w}^{(k)}}\right)
$$

where, $\phi(t, l)$ is a coefficient gives the strength of the BP learning, corresponds to the neighborhood function in SOM. $l$ is the distance of winner modules and learnt modules. It should be noted that $\phi(t, l)$ is monotonically decreasing by $l$. Therefore winner module learns the best, and distanced module from winner one learns less. In addition, $\phi(t, l)$ is a monotonically decreasing by $t$, therefore neighborhood function become less from the viewpoint of number of learning progress.

$\phi(t, l)$ in this learning is shown in equation (7). $\sigma(t)$ is a monotonically decreasing function decreasing with time $t$.

$$
\phi(t, l)=\exp \left(-\frac{l^{2}}{2 \sigma^{2}(t)}\right)
$$

Using a general mnSOM, it is impossible to expect the spatial interpolation because module will show similar input and output characteristics which are clustered by SOM learning with temporal projection progress. The reason why a general mnSOM cannot be used is because in general mnSOM the initial weights are set randomly to the ANN in a module and the one which has the closest characteristic will be the winner.

In the past research of the authors of this paper, it is confirmed that pre-training with the winner module which is the location of selected data group directly is effective for learning in which no spatial information is lost. We call this method "Projection-mnSOM (hereafter P-mnSOM)".

In P-mnSOM, the input and output data and learning algorithm are almost similar to general mnSOM. The difference between general mnSOM and P-mnSOM is on their determination method of winner module.

In general mnSOM, winner module is the one which has minimum error or average error in all modules. However in $\mathrm{P}-\mathrm{mnSOM}$, winner module location is the location of selected data group directly.

The decision algorithm of winner module (equation (4) in $\mathrm{mnSOM}$ ) is replaced with following equation (8). Actually this is the difference between general mnSOM and P-mnSOM.

$$
k_{\text {winner }}\left(D_{i}\right)=i
$$

By the replacement, we changed the module of minimum error to the module which is corresponded to the observatory location of selected input / output data. Furthermore, the module of observatory location has the characteristic of input / output data of own location and the winner module is determined regardless of initial weights set in ANN of a module.

After pre-training, we do the same learning process similar to general mnSOM. As the weights among neurons in ANN of module has the characteristic of own spatial location, the winner module is almost own observatory location which is decided by decision algorithm in general mnSOM. Therefore, the relation between module and observatory is kept.

\section{B. Grid-Style mnSOM}

In P-mnSOM, each observatory is mapped as a module in mnSOM. In this study, we integrated the entire actual geographical map into mnSOM so that we can predict more detail points between observation sites. By unify mnSOM and geographical map, the wind speed projection which location is not observatory becomes possible.

In the other word, the location of observatory is fit into the mnSOM which is composed of modules arranged on the grid. Learning of mnSOM is performed for whole modules, 
applicable to observatory and no observatory, the wind speed projection of the location which is not observatory (unknown data) becomes possible.

The concept of the simulation method applied unified mnSOM is shown in Fig. 4.

\section{Data Construction}

The purpose of this research is to project the wind speed of an arbitrary location by using the wind conditions in certain (surrounded) regions. Therefore, the data for learning mnSOM is wind speed and wind direction as input and output. Since it is important to use the spatial characteristics as input data and the teacher signal as output data, wind speed $v(t)$ and wind direction $\theta$ (angle) are taken into account. In order to handle the input and output data to wind speed and wind direction only by wind speed, the wind speed vector is divided to north-south and east-west elements based on the wind direction. As north wind direction is taken as 0 angle, the wind speeds of north-south direction and east-west direction can be presented by the following Fig. 5 .

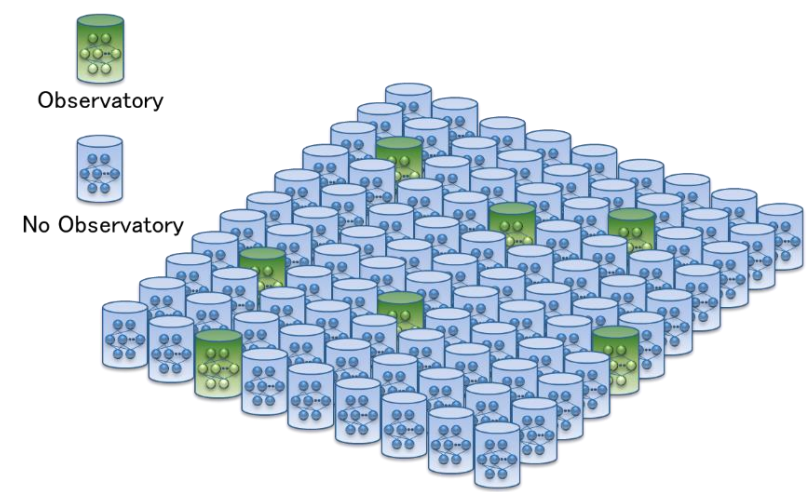

Fig. 4. Grid-style mnSOM.

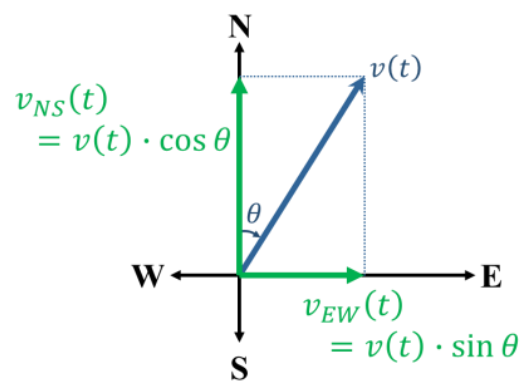

Fig. 5. Restructure of wind speed and direction.

Furthermore, the input data and teacher signal are translated to vectors by the reconstructed time delay coordinate system, based on deterministic chaos concept, for learning the dynamics of wind speed. The $\boldsymbol{x}$ as input vector is shown in equation (9) and the $\boldsymbol{y}$ as output vector is shown in equation (10). In these equation, $m$ is dimensions, $\tau$ is time delay in reconstructed dimension and $s$ is steps of projection. (The unit of $\tau$ is link to steps of projection. In the study, it is hour.)

$$
\begin{gathered}
\boldsymbol{x}=\left(v_{N S}(t), v_{E W}(t)\right), \ldots,\left(v_{N S}(t+(m-1) \tau), v_{E W}(t+(m-1) \tau)\right) \\
\boldsymbol{y}=\left(v_{N S}(t+s), v_{E W}(t+s)\right), \ldots,\left(v_{N S}(t+(m-1) \tau+s), v_{E W}(t+(m-1) \tau+s)\right)
\end{gathered}
$$

\section{Simulation}

For this research, a simulator is created which can simulate all necessary calculations including projection of wind speed as shown in Fig. 6.

We project the wind speed of Choshi site using the wind speed and wind direction data of Choshi (a) and 11 surrounded points from (b) to (l) as shown in Table I.

In this simulation, there are 11 observation sites inside the grid, only the weather data of closer 3 sites, Yokoshiba-hikari, Katori and Kashima is used. Then temporal and spatial projection of one hour ahead for Choshi is performed.

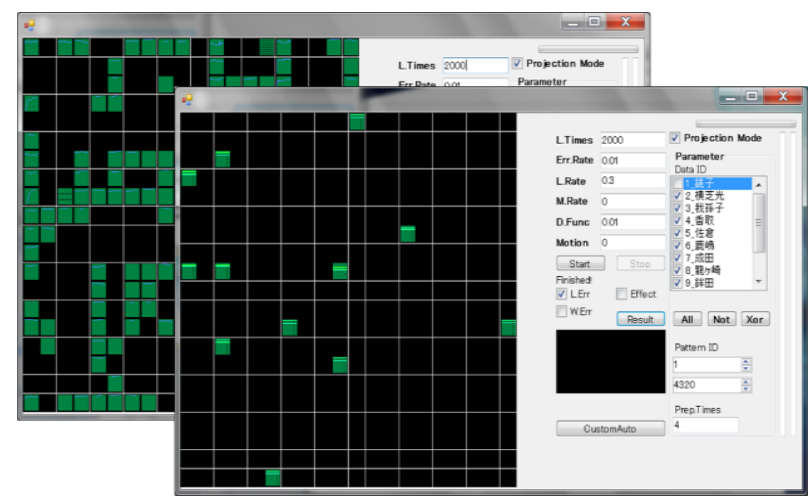

Fig. 6. Developed simulator.

TABLE I: OBSERVATORIES (CHOSHI AND THE NEAR SITES)

\begin{tabular}{lcccc}
\hline \hline & $\begin{array}{c}\text { North } \\
\text { latitude } \\
\text { [degree] }\end{array}$ & $\begin{array}{c}\text { East } \\
\text { longitude } \\
\text { [degree] }\end{array}$ & $\begin{array}{c}\text { Distance } \\
\text { from } \\
\text { Target } \\
{[\mathbf{k m ]}}\end{array}$ & $\begin{array}{c}\text { Direction } \\
(*) \\
\text { [degree] }\end{array}$ \\
\hline a) Choshi & 35.738 & 140.857 & - & - \\
\hline b) Yokoshiba-hikari & 35.657 & 140.480 & 35.255 & 255.291 \\
\hline c) Abiko & 35.863 & 140.110 & 68.864 & 281.876 \\
\hline d) Katori & 35.858 & 140.502 & 34.725 & 292.728 \\
\hline e) Sakura & 35.728 & 140.212 & 58.295 & 269.094 \\
\hline f) Kashima & 35.963 & 140.622 & 32.817 & 319.818 \\
\hline g) Narita & 35.763 & 140.385 & 42.733 & 273.872 \\
\hline h) Ryugasaki & 35.890 & 140.212 & 60.634 & 286.393 \\
\hline i) Hokota & 36.168 & 140.527 & 56.352 & 328.246 \\
\hline j) Tsuchiura & 36.103 & 140.220 & 70.346 & 305.468 \\
\hline k) Tsukuba & 36.057 & 140.125 & 74.954 & 298.493 \\
\hline 1) Shigehara & 35.417 & 140.310 & 61.070 & 234.348 \\
\hline \hline
\end{tabular}

(*) North is 0 degree, East is 90 degrees, South is 180 degrees, and West is 270 degrees.

The parameters of the simulation which are determined by trial-and-error are as follows:

- Dimensions : $m=2$

- Time Delay: $\tau=1$

- ANN Neurons : Input 4 - Hidden 8 - Output 4

- Learning Rate : 0.3

- Length of using data: $L=4320$

- Steps of projection: $s=1$

The number of hidden neurons and $\tau$ are important parameters for this simulation because of their impact on network construction and leading the model toward proper results. There are some methods for obtaining these parameters such as rule of thumbs, etc, however there is no general solution for this problem. In this study, based on the authors experience and many reported articles, a trial and error scheme is used as it proved to be more effective for determination of appropriate number of hidden neurons and $\tau$ 
Regarding the determination of hidden neurons, it is an art rather than mathematics. When the number of hidden neurons is small, the correlation of the output and input cannot be studied well and the error increase. Moreover, when the number of hidden neurons is more than the necessity, even an irrelevant noise is studied to the correlation of both, and therefore the error grows moreover in this case. Therefore, the selection of the hidden neurons is done by add a small number and then gradually increases the number and calculate the error until it reaches to the lowest error. In this study we started from 1 to 20 neurons, as a result we obtained the lowest error when the number of hidden neurons was 8 .

Similarly, regarding the determination of la $\tau$, we confirmed the error while changing the number of $\tau$ from 1 to 12 , and the best error was obtained when the number was 1 . We think the cause is correlation of the prediction step.

Finally, the simulations are evaluated based on the comparison of results found for projection of Choshi with the data of Choshi directly, and the results of projection of Choshi with the data from 3 near observatory points.

TABLE II: PROJECTION ERROR BY P-MNSOM AND GRID-STYLE MNSOM

\begin{tabular}{lcc}
\hline \hline & $\begin{array}{c}\text { RMS } \\
{[\%]}\end{array}$ & $\begin{array}{c}\text { Absolute Average } \\
\text { Error }[\mathbf{m} / \mathbf{s}]\end{array}$ \\
\hline P-mnSOM & 1.932 & 1.434 \\
\hline Grid-Style mnSOM & 1.622 & 1.142 \\
\hline \hline
\end{tabular}

\section{A. Projection with both P-mnSOM and Grid-Style mnSOM}

First, we compare the projection results determined by P-mnSOM and Grid-Style mnSOM in order to comfirm that Grid-Style mnSOM has accuracy of projection similar to P-mnSOM. In P-mnSOM, the number of modules for learning is 11 corresponding to observation sites. And in Grid-Style mnSOM, the number of modules is $16(4 \times 4)$ into which divided the area including 11 observation sites. The other parameters of simulation are same value.

The error of wind speed projection of Choshi with 3 area's weather data is evaluated using the RMS(Root Mean Square) error and Absolute Average error shown in Table II. The results are plotted in Fig. 7. The error of P-mnSOM and Grid-Style mnSOM is almost same.

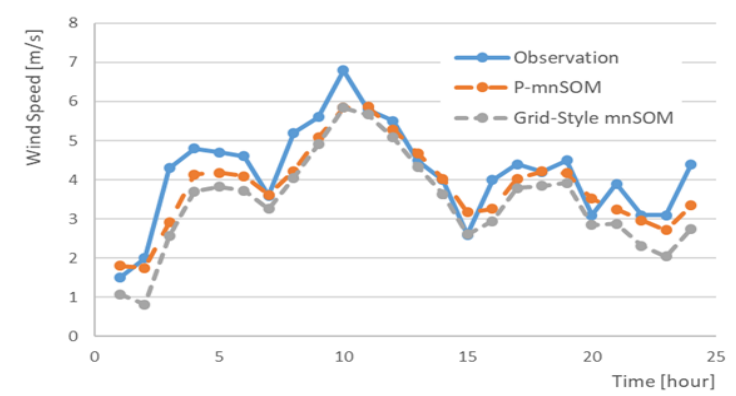

Fig. 7. Results of projection of P-mnSOM and grid-style mnSOM.

\section{B. Projection with Changing the Grid Size}

Second, we project wind speed by using the Grid-Style mnSOM by changing the grid size from $16(4 \times 4)$ to $100(10$ $\times 10$ ), and the other parameters of simulation are same value. The result error of projection is shown in Table III. The better results of top 3 are shown in Fig. 8. As the grid size gets bigger, the result error is increasing. We considered that the learning became difficult by making the network of mnSOM more complicated.

TABLE III: PROJECTION ERROR WITH GRID SIZE

\begin{tabular}{ccc}
\hline \hline $\begin{array}{c}\text { Grid Size } \\
\text { (Count of Module) }\end{array}$ & $\begin{array}{c}\text { RMS } \\
{[\%]}\end{array}$ & $\begin{array}{c}\text { Absolute Average } \\
\text { Error }[\mathbf{m} / \mathbf{s}]\end{array}$ \\
\hline 16 Modules & 1.622 & 1.142 \\
\hline 25 Modules & 1.700 & 1.216 \\
\hline 36 Modules & 1.711 & 1.247 \\
\hline 49 Modules & 1.970 & 1.494 \\
\hline 64 Modules & 1.813 & 1.408 \\
\hline 81 Modules & 2.061 & 1.558 \\
\hline 100 Modules & 2.151 & 1.648 \\
\hline
\end{tabular}

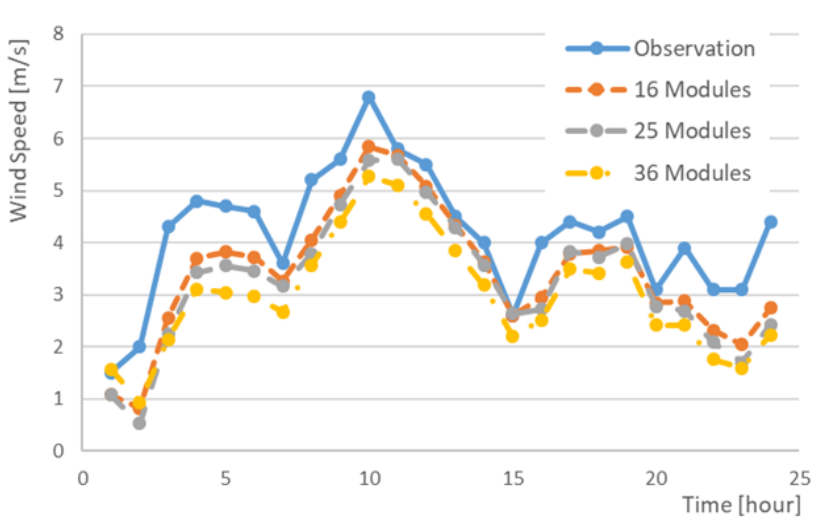

Fig. 8. Results of projection by changing grid size.

TABLE IV: PROJECTION ERROR WITH GRID SIZE AND LEARNING COUNT

\begin{tabular}{cccc}
\hline $\begin{array}{c}\text { Grid Size } \\
\text { (Count of Module) }\end{array}$ & $\begin{array}{c}\text { Learning } \\
\text { Count }\end{array}$ & $\begin{array}{c}\text { RMS } \\
{[\%]}\end{array}$ & $\begin{array}{c}\text { Absolute Average } \\
\text { Error [m/s] }\end{array}$ \\
\hline 16 Modules & 10000 & 1.622 & 1.142 \\
\hline 25 Modules & 15625 & 1.630 & 1.191 \\
\hline 36 Modules & 22500 & 1.432 & 1.033 \\
\hline 49 Modules & 30625 & 1.373 & 0.963 \\
\hline 64 Modules & 40000 & 1.500 & 1.117 \\
\hline 81 Modules & 50625 & 1.315 & 0.918 \\
\hline 100 Modules & 62500 & 1.934 & 1.524 \\
\hline \hline
\end{tabular}

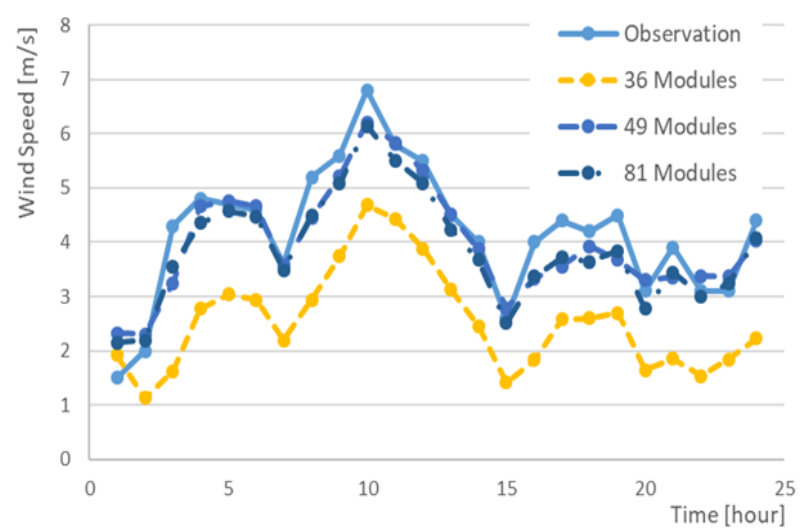

Fig. 9. Results of Projection with Fitting the Learning Count.

\section{A. Projection with Fitting the Learning Count to Grid Size}

Finally, we project wind speed by changing the learning count to fit to grid size. By the result of simulation by changing the grid size only, we found that more errors resulted in more module. So we adjusted the learning count to be the same for each module.

The result error of projection is shown in Table IV, and the main results of projection are shown in Fig. 9. The best projection result is when the grid size is 49 or 81 modules. And by adjusting the learning count, even if the grid size 
changes, the projection error does not change much.

\section{CONCLUSION AND REMARKS}

Wind power generation is an effective source for solution of environmental problems, and it is in progress in many parts of the world. For an island country like Japan, it is expected to obtain a stable wind power generation in off-shore rather than on-shore. It is important to choose a right place with high wind speed since it is directly related to profitability. However, comparing with the on-shore, the off-shore sites have some problems regarding the technical and cost which need to be investigated carefully.

Therefore, the purpose of this research was to develop an approach to project the unknown wind speed of site, where is distant from observation points, more detailed and more precise based on the observation of weather information around the target off-shore site. By the Grid-Style mnSOM developed in this study, it became possible to reduce the risk related to prior weather research in the introduction point examination of off-shore wind power generation more.

In this research, we further develop P-mnSOM which had already confirmed its effectiveness. And the projection of more detailed and more precise was examined successfully by arranging the modules in Grid-Style.

Since we don't have the weather data of off-shore site, in this research, we used the weather data of Choshi and 3 near observatory points to project the wind conditions for Choshi and evaluated for errors by comparing the actual observations data.

First, we compared the projection results which were determined by P-mnSOM and Grid-Style mnSOM. The results of Grid-Style mnSOM is almost as same as the one of P-mnSOM. It can be stated that Grid-Style mnSOM is major projection technique as $\mathrm{P}-\mathrm{mnSOM}$, that is for actual off-shore projections can successfully project the wind speed of off-shore from the observation point of the on-shore. Furthermore Grid-Style mnSOM is able to project the wind speed more detail point than P-mnSOM. So it is considered to be effective for predicting one specific point as in the offshore case.

Next, we confirmed the relationship of projection error with grid size (it is the same as module count). With module count is near to the number of P-mnSOM case, the error is not so different. But module count is increased, the projection error is higher. We considered the reason the relationship that learning count per each module is decreased because module count is increased and learning count is not changed.

So ultimately, we confirmed the changes in projection errors when learning count is fit to grid size. the learning count was determined by assuming that the number of learning per a module is 625 . The projection error was decreased in each grid size, so we confirmed that we can make further detailed prediction by dividing the grid finely.

In future actions, we will project the wind condition of an off-shore area with this method. Next, by projection the wind condition in a certain off-shore area, we will identify the best point suitable for wind power generation in that off-shore area.

\section{REFERENCES}

[1] IPCC TAR WG1. (2013). Stocker, T. F., D. Qin, G.-K. Plattner, M Tignor, S. K. Allen, J. Boschung, A. Nauels, Y. Xia, V. Bex, and P. M. Midgley., ed., "Climate change 2013: The physical science basis. Contribution of working group I to the fifth assessment report of the intergovernmental panel on climate change", Cambridge University Press

[2] Global Wind Energy Council, "Global wind report 2016 annual market update," Global Wind Energy Council, April 2017

[3] New Energy and Industrial Technology Development Organization, "White Paper on technology about renewable energy," Morikita Publishing Co., Ltd., Chapter 3, December, 2013, (in Japanese)

[4] M. Hayashi and K. Nagasaka, "Wind speed prediction and determination of wind power output with multi-area weather data by deterministic chaos," ICAMechS 2014, August 2014.

[5] M. Hayashi and K. Nagasaka, "Temporal and spatial projection of wind speed based on modular network som for installation of off-shore wind generation," ICAMechS 2015, August 2015.

[6] K. Tokunaga, T. Furukawa, and S. Yasui, "Modular network SOM self organization of a group of function models," Technical Report of IEICE NC2002-130, pp. 79-83. February, 2003.

[7] K. Tokunaga, T. Furukawa, and S. Yasui, "Modular network SOM self-organizing map of a systems group in function space," Technical Report of IEICE NC2003-17, pp. 31-36, June, 2003.

[8] K. Kimotsuki, K. Tokunaga, S. Yasui, and T. Furukawa, "Modular network SOM self-organizing maps dealing with dynamic systems," Technical Report of IEICE NC2003-141, pp. 35-40, March, 2004.



Mitsuharu Hayashi received his master's degree in electronic and information engineering from Tokyo University of Agriculture and Technology, Japan in 2003 Currently he is working toward the Ph.D. program in the field of projection for renewable energy in the Department of Electronic and Information Engineering at the Tokyo University of Agriculture and Technology, Tokyo, Japan. His research mainly wind speed projection and simulation by intelligent method such as artificial neural network and deterministic chaos to promote the installation of wind power generation.

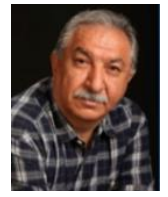

Ken Nagasaka obtained his $\mathrm{PhD}$ in electrical engineering from Tokyo Metropolitan University (1990). He became a chief researcher at CSD Company, Tokyo (1990-1991). He became a visiting professor at University of Manitoba, Winnipeg, Canada (1991-1994). He worked as a Scientist at Central Research Institute of Electric Power Industry, Tokyo (1994-1998). He became an associate professor at the Department of Electrical Engineering of Tokyo Metropolitan University (1995-1998). Since 1998 he taught and researched at the Graduate School of Engineering, Tokyo University of Agriculture and Technology. Currently he is a professor of Tokyo University of Agriculture and Technology. Prof. Nagasaka has published more than 600 papers in the area of power systems and renewable energies. 